\title{
Under the right conditions: protecting podocytes from diabetes-induced damage
}

Imran HA Ali and Derek P Brazil

See related research by Li et al., http://stemcellres.com/content/4/5/103
Mesenchymal stem cells (MSCs) are specialized progenitor cells that possess differentiation, proliferative and immunomodulatory potential. This, along with their ease of isolation, makes MSCs potentially an exciting new tool for regenerative medicine [5]. In this issue of Stem Cell Research \& Therapy, Li and colleagues show that conditioned media of MSCs derived from human adipose tissue (hAD-MSCs) significantly reduced the apoptosis of HGexposed podocytes. The key ingredient in this conditioned medium is suggested by the authors to be epidermal growth factor (EGF). Previous data from others suggested a potential beneficial effect of MSCs in the treatment of acute renal ischemia and DN $[5,6]$. hAD-MSCs injected into diabetic rats caused a reduction in DN severity without accumulation of MSCs within the kidneys [5]. This group therefore hypothesized that hAD-MSCs protected the kidney in a paracrine manner [5]. The potential of conditioned medium from hAD-MSCs to elicit a similar effect was assessed in the current study.

$\mathrm{Li}$ and colleagues used mouse podocyte clone 5 to elucidate the mechanism by which MSCs rescue HG-induced apoptosis of podocytes [1]. To establish a model of HGinduced podocytic apoptosis in vitro, the authors exposed the cells to normal glucose, normal glucose + mannitol, and HG for 24, 48 and 72 hours. An increase in podocyte apoptosis within the HG group at all time points was detected compared with the normal glucose and normal glucose + mannitol groups. This effect correlated with a decrease in the expression of podocyte proteins synaptopodin and nephrin within the HG group at all time points, suggesting that HG damaged the integrity of the glomerular filtration barrier.

Having established a model of HG-induced podocyte apoptosis, Li and colleagues next added MSC conditioned medium (MSC-CM) or human embryonic lung cell conditioned media (Wi38-CM) to the podocytes in all three treatment groups. The addition of MSC-CM decreased podocyte apoptosis and maintained the levels of synaptopodin and nephrin expression in the HG group.
* Correspondence: d.brazil@qub.ac.uk

Centre for Vision and Vascular Science, Queen's University Belfast, ICS-A, Grosvenor Road, Belfast BT12 6BA, UK 
The addition of Wi38-CM had no beneficial effect in this case. To understand why MSC-CM was more effective than Wi38-CM, Li and colleagues identified several cytokines that were present at higher levels in the MSC-CM compared with Wi38-CM. Of these, EGF was secreted in the greatest quantity. Given the previous reports of EGFs ability to repair and protect epithelial cells from apoptosis [7-9], the authors then treated podocytes exposed to HG with either MSC-CM or recombinant EGF. Flow analysis AnnexinV/Pi staining and western blot detection of caspase 3 confirmed the ability of EGF to inhibit HGinduced podocytic apoptosis. This effect was diminished when MSC-CM was co-incubated with a neutralizing antibody against EGF. This study clearly points to EGF as a key player within the MSC-CM in inhibiting HG-induced podocyte damage and apoptosis.

A particularly interesting aspect of this study is the demonstration that EGF plays a critical role in the restoration of the glomerular filtration barrier. EGF has been previously shown to promote the survival of epithelial cells of the intestine and kidney $[7,9]$. In contrast to the current study, others have shown a pathogenic role for the EGF receptor in DN [10] and progressive glomerulonephritis [11]. The current study clearly shows a cytoprotective role of EGF on podocytes exposed to HG. Further study is required to fully elucidate the disparity around the role of EGF in kidney disease, and also to more fully define the protective mechanism triggered by the conditioned medium from hAd-MSCs for podocytes during DN.

The work here augments seminal work done by others and identifies a tantalizing new possibility whereby injecting MSCs themselves for the treatment of DN (and indeed other diseases) may not be necessary. Rather, harvesting and injection of the conditioned medium from these cells may be sufficient to harness the potential needed to regenerate and repair damaged glomerular filtration membrane. Future work is needed to define the full potential of this novel cell-free treatment modality for $\mathrm{DN}$.

\footnotetext{
Abbreviations

DN: Diabetic nephropathy; EGF: Epidermal growth factor; hAD-MSC: Mesenchymal stem cell derived from human adipose tissue; HG: High glucose; MSC: Mesenchymal stem cell; MSC-CM: Mesenchymal stem cell conditioned medium; Wi38-CM: Human embryonic lung cell conditioned media.
}

\section{Competing interests}

The authors declare that they have no competing interests.

Published: 02 Oct 2013

\section{References}

1. Li D, Wang N, Zhang L, Hanyu Z, Xueyuan B, Fu B, Shaoyuan C, Zhang W, Xuefeng S, Li R, Chen X: Mesenchymal stem cells protect podocytes from apoptosis induced by high glucose via secretion of epithelial growth factor. Stem Cell Res Ther 2013, 4:103.

2. Mogensen CE, Christensen CK, Vittinghaus E: The stages in diabetic renal disease with emphasis on the stage of incipient diabetic nephropathy. Diabetes 1983, 32:64-78.
3. Xu ZG, Ryu DR, Yoo TH, Jung DS, Kim JJ, Kim HJ, Choi HY, KIM JS, Adler SG, Natarajan R, Han DS, Kang SW: P-Cadherin is decreased in diabetic glomeruli and in glucose-stimulated podocytes in vivo and in vitro studies. Nephrol Dial Transplant 2005, 20:524-531.

4. Eid AA, Gorin Y, Fagg BM, Maalouf R, Barnes JL, Block K, Abboud HE: Mechanisms of podocyte injury in diabetes: role of cytochrome P450 and NADPH oxidases. Diabetes 2009, 58:1201-1211.

5. LV SS, Liu G, Wang JP, Wang WW, Cheng J, Sun AL, Liu HY, Nie HB, Su MR, Guan GJ: Mesenchymal stem cells transplantation ameliorates glomerular injury in streptozotocin-induced diabetic nephropathy in rats via inhibiting macrophage infiltration. Int Immunopharmacol 2013, 17:275-282.

6. Tögel F, Hu Z, Weiss K, Isaac J, Lange C, Westenfelder C: Administered mesenchymal stem cells protect against ischemic acute renal failure through differentiation-independent mechanisms. Am J Physiol Renal Physiol 2005, 289:F31-F42

7. Sheng G, Guo J, Warner BW: Epidermal growth factor receptor signaling modulates apoptosis via p38alpha MAPK-dependent activation of Bax in intestinal epithelial cells. Am J Physiol Gastrointestin Liver Physiol 2007, 293:G599-G606.

8. $\quad$ Ying WZ, Zhang HG, Sanders PW: EGF receptor activity modulates apoptosis induced by inhibition of the proteasome of vascular smooth muscle cells. J Am Soc Nephrol 2007, 18:131-142.

9. Lu C, Ren W, Su XM, Chen JQ, Wu SH, Zhou GP: EGF-recruited JunD/c-fos complexes activate CD2AP gene promoter and suppress apoptosis in renal tubular epithelial cells. Gene 2009, 433:56-64.

10. Advani A, Wiggins KJ, Cox AJ, Zhang Y, Gilbert RE, Kelly DJ: Inhibition of the epidermal growth factor receptor preserves podocytes and attenuates albuminuria in experimental diabetic nephropathy. Nephrology (Carlton) 2011, 16:573-581.

11. Bollee G, Flamant M, Schordan S, Fligny C, Rumpel E, Milon M, Schordan E, Sabaa N, Vandermeersch S, Galaup A, Rodenas A, Casal I, Sunnarborg SW, Salant DJ, Kopp JB, Threadgill DW, Quaggin SE, Dussaule JC, Germain S, Mesnard L, Endlich K, Boucheix C, Belenfant X, Callard P, Endlich N, Tharaux PL: Epidermal growth factor receptor promotes glomerular injury and renal failure in rapidly progressive crescentic glomerulonephritis. Nat Med 2011, 17:1242-1250.

$10.1186 /$ scrt330

Cite this article as: Ali and Brazil: Under the right conditions: protecting podocytes from diabetes-induced damage. Stem Cell Research \& Therapy 2013, 4:119 